\title{
The Moderating Effects of Perceived Emotional Benefits on Inhalant Initiation Among American Indian and White Youth
}

\author{
Randall C. Swaim, PhD \\ Department of Psychology, Tri-Ethnic Center for Prevention Research, Colorado State University, \\ Fort Collins, Colorado
}

\begin{abstract}
Background-Inhalant use co-occurs with emotional distress. Inhalant use may be a means of self-medicating distress, but more recent study focuses on the cognitive appraisal of personal benefits of using substances.
\end{abstract}

Objectives-Objectives were to determine whether emotional distress variables predict early versus later initiation of inhalant use, whether such relationships differ between American Indian and white youth, and whether perceived emotional benefits of inhalant use moderates the relationship between emotional distress and stage of inhalant initiation.

\begin{abstract}
Methods-Data were from a study of 7-12th grade American Indian youth who live on or near reservations. A total of 856 students from 32 schools surveyed from 2009 to 2012, who reported having used inhalants (American Indian $=683$; white $=173$ ), were surveyed about age first use of inhalants, levels of emotional distress, and perceived benefits of inhalant use. SEM models were used to assess study objectives.
\end{abstract}

Results-Depression and anger did not discriminate between early and later initiation. Lower self-esteem related to earlier initiation, but only among American Indian students. Perceived emotional benefits of inhalant use did not moderate the relationship between self-esteem and stage of initiation.

Discussion and Conclusions-Among middle school and high school American Indian and white youth living on or near American Indian reservations, emotional distress is not strongly related to stage of inhalant initiation.

Scientific Significance-These findings raise questions about the timing and strength of relationship between emotional distress and early inhalant initiation. Prospective studies are need to assess this relationship more fully.

\section{INTRODUCTION}

The comorbidity among substance use and emotional factors is well established. Substance use often co-occurs with various negative emotional states including depressed or anxious

\footnotetext{
Address correspondence to Dr. Swaim, Tri-Ethnic Center for Prevention Research, Colorado State University, 106 Sage Hall, Fort Collins, CO, 80523-1876. Randall.Swaim@Colostate.Edu.

Declaration of Interest

The author reports no conflicts of interest. The author alone is responsible for the content and writing of this article.
} 
mood, agitation, or anger, or low self-esteem, ${ }^{1}$ and emotional distress factors have been found to predict different trajectories of adolescent substance use. ${ }^{2}$ Beyond the effects of emotional distress on adolescent substance use in general, there is substantial evidence that this relationship is particularly prevalent for inhalant use. One source of distress, low selfesteem, discriminates between users and non-users of inhalants among troubled youth. ${ }^{3}$ Both adult ${ }^{4,5}$ and adolescent ${ }^{6,7}$ inhalant users also tend to be more depressed than their nonusing counterparts, and there is a higher prevalence of depressive and anxiety disorders for adult inhalant users. ${ }^{4,5}$

One theoretical approach to understanding the relationship between emotional states and substance-using behaviors is the self-medication hypothesis (SMH) of Khantzian ${ }^{8}$ which postulates that use of various psychoactive substances serves to modulate and control distressful emotions. But this theory has not been without its critics. ${ }^{9}$ Whereas SMH is typically presented as a motivational theory with its roots focused on psychoanalytic processes, other more recent models focus on the cognitive appraisal of substances as sources of psychological benefit. Such models include self-efficacy and outcome expectancies ${ }^{10,11}$ and perceived risks and benefits ${ }^{12,13}$ as targets of cognitive appraisal. The Theory of Normative Social Behavior ${ }^{14}$ (TNSB) postulates that outcome expectancies, the belief that a behavior (eg, substance use) will lead to a certain outcome, may serve as a moderating factor on the relationship between descriptive norms and behavioral intentions. In relation to $\mathrm{SMH}$, outcome expectancies for specific emotional outcomes should moderate the relationship between emotional states and substance use behaviors. The relationship between emotional states and substance use should be strengthened if subjective appraisal of the substance is viewed as beneficial for emotional control and benefit.

In this paper I evaluate the moderating effects of perceived emotional benefits on the relationship between self-esteem, anger, and depression and early initiation of inhalant use among American Indian and white youth attending schools on or near American Indian reservations. Early initiation of inhalant use in general population samples increases risk for mood, anxiety, and personality disorders. ${ }^{5}$ In a comparison of 3480 American Indian and white youth living on or near reservations, Native youth were significantly more likely to initiate inhalant use compared to white youth (Stanley LR, Swaim RC, unpublished data, January, 2014; $O R=1.76$ ). A common approach to investigating substance use onset is to dichotomize samples into early versus later initiation. ${ }^{15}$ In this study a cut point of age 12 and younger for early initiation was selected, as this age break relates to a transition from elementary to middle school when substance use risk is increased. As the racial/ethnic adolescent group that consistently reports the highest levels of substance use, ${ }^{16}$ a clearer understanding of the relationship between emotional distress and substance use behaviors is needed for American Indian youth.

We hypothesize first, that emotional factors (self-esteem, depression, anger) will be more highly related to early than to later initiation of inhalant use. This is based on prior studies that show increasing levels of emotional distress and mental health disorders among early inhalant initiators. ${ }^{3-5}$ While a specific hypothesis cannot be made regarding the relationship between emotional factors and inhalant initiation based on ethnicity, the following is raised as a research question. Do various forms of emotional distress relate to stage of inhalant 
initiation differentially between American Indian and white youth? Finally, it is hypothesized that perceived emotional benefits will moderate the relationship between emotional factors and inhalant initiation for both groups of youth.

\section{METHOD}

\section{Participants}

The participants included 7th through 12th grade youth who attended schools on or near American Indian reservations. This study is part of a larger ongoing epidemiologic study of substance use among reservation-based Native youth. To be included in the study, the schools had to have at least 20\% American Indian youth enrolled. Sampling of schools for the larger project was based on obtaining a representative sample from six geographic regions (Northwest, Northern Plains, Southeast, Southern Great Plains, and Southwest). Schools were paid $\$ 500$ for participation and were provided with a comprehensive report of survey findings. The specific tribal identity of youth is kept private for purposes of confidentiality.

The sample contained four years of data (2009-2012 school years) from 32 schools. Students who reported at least lifetime use of inhalants were included in the study and were divided into early initiation (12 or younger) and later initiation (13 or older) groups. Ethnic identification was based on self-report by the students, and two groups of students, American Indian and white, were included. A total of 683 American Indian students reported having used inhalants (19.9\%). Of this number, 347 were early initiators and 336 were later initiators. A total of 173 white students reported having used inhalants (9.9\%), with 71 early initiators and 102 later initiators.

\section{Procedure}

The data collection procedures were approved by the university Institutional Review Board (IRB) and a resolution of support was also obtained from tribal authorities or school board prior to all data collection. A teacher or school staff member was trained in human subjects procedures through an online or telephone IRB training protocol prior to survey administration. The school staff member carried out all survey administration following written instructions. Parents were notified of survey administration through both a parent notification letter and a broad media release and were given the opportunity to remove their child from the project. Less than one percent of students did not complete the survey due to lack of parental consent

Students were informed that their participation was voluntary and that they could leave any survey item blank, or discontinue taking the survey at any time. No identifying information was collected to maintain the anonymity of all participants. Surveys were completed during one class period and those students for whom parental consent was not obtained were moved to another school area. After surveys were completed, students placed their completed survey in a large envelope in random order which was then sealed and turned in to the school staff member. Staff administering the surveys were instructed to remain in an area of 
the classroom during survey completion that precluded their observation of student responses.

\section{Measures}

Survey data were obtained from The American Drug and Alcohol Survey ${ }^{\mathrm{TM}}$. Versions of this survey have been given to American Indian youth for several decades, and it has been validated for use with both majority and ethnic minority youth. ${ }^{17}$ Two inhalant use items, one assessing lifetime use and the other assessing age of first use, were utilized. The lifetime item asked, "Have you ever "sniffed" or "huffed" glue, gas, sprays, or anything like that to get high?" The second item asked, "How old were you the first time you "sniffed" or "huffed"? Response alternatives for this item included, "never used", 7 or younger, individual alternatives from age 8 to 18 , and 19 or older.

Measures of emotional distress included items assessing level of self-esteem, depression, and anger. These measures have been used successfully in several previous studies with both majority and minority youth, with evidence of good psychometric properties. ${ }^{18-21}$ Each of the emotional distress items was measured on a four-point Likert scale with responses of "a lot", "some", "not much", and "not at all." The self-esteem measure was comprised of six items (eg, "I am proud of myself," "I am good looking"; Cronbach alpha = .81). Legitimate and important questions have been raised as to the best way to measure self-esteem among American Indian samples ${ }^{22}$ considering the emphasis on collective as opposed to personal aspects of self-concept among many American Indian cultural groups. ${ }^{23}$ However, both collective and personal measures of self-esteem have been used successfully with Native people, ${ }^{22}$ and personal measures have demonstrated measurement equivalence between American Indian and non-Indian youth. ${ }^{24}$ The self-esteem measure reported here, as seen in the example items cited above, is a personal measure that has been used successfully with American Indian youth, ${ }^{25,26}$ and has demonstrated construct validity with Native samples. ${ }^{27}$ The depression measure was assessed with five items (eg, "I feel low," "I am depressed"; Cronbach alpha $=.89$ ). The anger measure was comprised of four items (eg, "I am quicktempered," "I get mad"; Cronbach alpha $=.80$ ).

The emotional benefits of inhalants measure was comprised of three items following the stem, "Sniffing inhalants ..." Items included "makes me feel good," "is fun," and "helps me feel better (Cronbach alpha $=.99$ ).

\section{Missing Data Management}

Missing data were handled using a multiple imputation approach. ${ }^{28}$ Five imputed data sets were created using IBM SPSS Statistics 22, and parameter estimates were combined using procedures outlined by Rubin. ${ }^{29}$ There is no known theoretical approach for combining fit statistics within a multiple imputation procedure for SEM. Therefore, Chi-square and other measures of fit were reported using the mean across the five imputations.

\section{Analytic Approach}

Mean levels of emotional distress variables were compared between early and later inhalant initiation for both American Indian and white adolescents with independent $t$-tests. This was 
followed up with evaluation of both measurement and structural models within an SEM framework using EQS 6.1. ${ }^{30}$ Measurement models were constructed to evaluate the measurement structure of the three emotional distress variables with variances fixed to 1 and all factors allowed to freely correlate. This was followed by structural models in which stage of inhalant initiation (early or later) was evaluated for association with measures of emotional distress (depression, anger, low self-esteem). Multiple group models were assessed to determine first, whether the latent constructs for emotional distress were measured equivalently across groups. Four groups were assessed: (1) American Indian youth reporting high emotional benefits, (2) American Indian youth reporting low emotional benefits, (3) white youth reported high emotional benefits, and (4) white youth reporting low emotional benefits. The multiple group models were run first with no equality constraints imposed. This was followed by a model with all factor loadings constrained to be equal. Factor loading equivalence was assessed using a Chi-square difference test. A similar procedure was followed for the structural model, first with structural paths from emotional distress variables to stage of inhalant initiation freely estimated, followed by imposition of equality constraints on the structural paths. A test for equivalence of structural paths was again assessed with a Chi-square difference test. Robust estimates of Chi-square and CFI, ${ }^{31}$ along with the SRMR were used to evaluate model fit. Non-normality of distributions was accounted for using Robust estimation in EQS which provides adjusted standard errors, and the Satorra-Bentler (S-B) Chi-square is reported. For Chi-square difference tests, the standard Chi-square was used. Standards for good model fit were based on a CFI near .95, and an SRMR less than $.09,{ }^{32}$ though others have questioned the meaningful differences between CFI's of .90 versus $.95 .{ }^{33}$ The structural model is presented in Figure 1 with moderating effects of emotional benefits depicted as arrows to regression paths from emotional distress variables to inhalant initiation.

\section{RESULTS}

Among American Indian students, $19.9 \%$ reported having ever used inhalants compared to 9.9\% of white students. Mean levels and standard deviations of emotional distress variables by ethnicity and stage of inhalant initiation are presented in Table 1. Independent t-tests across levels of inhalant initiation revealed one significant difference among the three emotional distress variables. Students who reported using inhalants at age 12 or younger reported lower levels of self-esteem than those who started using after age 12 (17.76 vs. $18.64 ; t(855)=3.141, p=.002)$. Follow-up tests showed that this difference occurred only among American Indian students, $t(681)=2.25, p=.025)$. With comparisons across ethnicity within stage of initiation, no differences were found among youth who began using inhalants earlier. Among those who initiated inhalants at age 13 or older, white students reported higher levels of anger compared to American Indian students, $t(368)=1.98, p=$. 048.

For assessment of latent variable models, five imputations were conducted. Measurement models were evaluated for each ethnicity for the three emotional distress constructs. Mean fit statistics across the five imputations for white students were, S-B Chi-square (87) = $185.757, p<.001, \mathrm{SRMR}=.090$, Robust CFI $=.89$. For American Indian students mean fit statistics were, S-B Chi-square $(87)=490.815, p<.001$, SRMR $=.071$, Robust CFI $=.89$. 
Factor loadings and structural coefficients across the four groups are presented in Table 2. All factor loadings were significant $(p<.001)$ based on robust standard errors with the exception of one indicator for self-esteem ("I am good at games") among white students reporting low emotional benefits $(p<.01)$. Only one structural coefficient was significant, from self-esteem to stage of inhalant initiation among American Indian students who perceived high emotional benefit of inhalants $(p<.05)$. With all factor loadings freely estimated for the measurement model, Chi-square $(\mathrm{df}=348)=1158.271$. With factor loadings constrained to be equal, Chi-square $(\mathrm{df}=393)=11.93 .426$. The Chi-square difference test, Chi-square $(\mathrm{df}=45)=35.155$, was non-significant $(p=.85)$, indicating that each of the measures of emotional distress was measured equivalently across the four groups. With all structural coefficients freely estimated, Chi-square $(\mathrm{df}=432)=1248.156$. With all structural paths constrained to be equal, Chi-square $(\mathrm{df}=441)=1249.956$. The Chi-square difference test, Chi-square $(\mathrm{df}=9)=1.80$, was non-significant $(p=.99)$, indicating that there were no moderating effects of perceived emotional benefits on the relationship between emotional distress factors and stage of inhalant initiation.

\section{DISCUSSION AND CONCLUSIONS}

In the current sample, $10 \%$ of white youth and $20 \%$ of American Indian youth reported having ever used inhalants. It is important to remember that these youth attended the same schools on or near American Indian reservations, and thus were subject to some of the same socio-economic conditions. Given that inhalant use increases risk for subsequent other substance use disorders including IV opiate use, ${ }^{5}$ the levels of use among this sample of youth, and the wide discrepancy in rates across these two cultural groups underscores the public health importance of this issue and the importance of identifying key etiologic mechanisms.

One potential explanation for the reported relationship between inhalant use and poor emotional and substance use outcomes is self-medication. ${ }^{8}$ Users are presumed to utilize illicit substances to treat their negative emotional states. It is also reasonable to suspect that those who expect higher levels of emotional benefit from such substance use would be more likely to use those substances and to begin using them at an earlier age if the negative emotional states are active. To test the validity of these questions, we evaluated two hypotheses and one research question. The first hypothesis predicted that emotional distress factors (low self-esteem, depression, and anger) would be related to early versus later initiation of inhalant use. Secondly, we asked the research question, whether American Indian students' emotional distress would be more strongly related to early inhalant initiation compared to white students. Finally, we considered a second hypothesis that perceived emotional benefits from using inhalants would moderate the relationship between emotional distress factors and early initiation of inhalants.

Little support was found for the first hypothesis. There was no strong relationship between early versus late inhalant initiation and emotional factors, with one exception. American Indian students with lower self-esteem generally were more likely to start using inhalants early. The other two emotional distress factors, depression and anger, were not associated with stage of inhalant initiation. As noted earlier, there is strong support in the literature for 
the likelihood of negative emotional outcomes among youth who start use of illicit substances at an early age. ${ }^{3-6}$ These findings support and extend those of Howard and Jensen $^{3}$ who found lower self-esteem among users compared to non-users of inhalants. These results extend their findings, indicating that lower self-esteem not only differentiates users and non-users, but also discriminates between early versus later initiators of inhalants. However, the effect was not strong. The failure to find support for a relationship between depression and inhalant initiation would appear to weaken the self-medication hypothesis. Several studies have found high levels of depression among inhalant users compared to nonusers. ${ }^{4-7}$ But depression did not discriminate between early and later inhalant initiators. Nor did levels of anger differentiate these groups.

In regard to the finding for self-esteem, it is important to note that the measure used here was based on personal self-esteem, an approach that has been used in most prior studies among American Indian youth. ${ }^{22,25,26}$ But given the potential important role of this construct in the emergence of early inhalant use, more study should consider the comparative effects of personal vs. collective self-esteem as reported by Hammond et al. ${ }^{35}$

Investigation of the research question revealed few differences in emotional factors by ethnicity. While there was an overall increased vulnerability for early inhalant use related to low self-esteem, follow-up tests showed that this relationship was present only for American Indian youth. No differences were found across ethnicity for depression. A somewhat surprising effect was found for anger, however, with white students reporting higher levels than American Indian students, among those who initiated inhalant use at age 13 or older.

The last question evaluated was whether perceived emotional benefits from inhalant use would moderate the relationship between emotional distress factors and stage of inhalant initiation. With comparison of structural coefficients across the four groups of youth, no moderating effects were found. An examination of the structural coefficients suggests that limited power may have suppressed some effects. Among white students, the raw value of coefficients from self-esteem to inhalant initiation equaled or exceeded those found among American Indian students, but were non-significant due to adjusted standard errors for distributional properties. Sample sizes for white students were considerably smaller. Future study that includes larger numbers of white students may support a finding for self-esteem and inhalant initiation.

A limitation that must be considered in the interpretation of these findings is the level of model fit. None of the models reached the preferred level of fit (CFA 2.95). Models were at or near the level of .90 , a standard that has been regarded as approximate fit. ${ }^{33}$ It is possible that with the inclusion of other possible measures of emotional distress (eg, anxiety), fit might have been improved. Inclusion also of correlated error terms would have improved fit, but would have made the interpretation of the latent variables less clear, so they were not included. The standard for SRMR was met. Therefore, fit was judged to be sufficient at or near .90 , making interpretation of findings appropriate.

While the current data cannot establish causal relationships, a number of issues are raised by these findings. The limited support for hypothesis one, that emotional distress would be 
more highly associated with earlier inhalant initiation, would appear to be in conflict with other findings that show early initiation to be associated with various mood, anxiety, and other disorders. ${ }^{5}$ But the results reported here suggest that the role of emotional factors may not be substantial in leading to early inhalant use. While past study has shown that inhalant users evidence high rates of mood, anxiety, and personality disorders ${ }^{5}$, this relationship may not be evident or active at early ages. Rather, emotional distress and for some, diagnosable psychiatric conditions, may emerge with continued use or as inhalant users move on to using other substances as reported by Novins and Barron ${ }^{36}$ and Storr, Westergaard, and Anthony. ${ }^{37}$

Other research ${ }^{3}$ has shown that those who develop an inhalant use disorder also show evidence of antisocial behaviors and early onset violence. But the data from this study showed no relationship between anger and early onset of inhalant use. This again suggests that whatever relationship exists between inhalant use and externalizing behaviors may not be clearly identifiable among school-age users.

Further work is needed to determine how emotional distress, and mood and behavior disorders relate to inhalant use. These findings raise questions as to whether early distress is causally related to early initiation of substance use among both American Indian and white adolescents. This may suggest that emotional factors, including later diagnosis of psychiatric conditions, may be outcomes of inhalant use, or that the early precursors of causally related emotional factors are not yet detectable among adolescents.

The current study was limited by its cross-sectional design, but it is as least partially in contradiction to a number of past studies. This introduces new questions regarding the etiology and outcomes associated with inhalant use. Due to the retrospective nature of one variable, age of onset, it is possible that other factors intervened between onset and the reporting of current perceived emotional distress. Other factors could have diminished earlier emotional distress, or some who tried inhalants may not have been persistent users, reducing the emotional distress relationship for the entire sample. While these limitations need to be considered, the results reported here emphasize the need for prospective studies that follow youth from early adolescence into young adulthood to help clarify the role of emotional distress and inhalant use trajectories, with identification of potentially causal mechanisms of early onset. Furthermore, while only one ethnicity difference was found, regarding self-esteem among American Indian students, this findings underscores the importance of also considering potentially differing relationships across ethnic groups of youth in future work in this area.

\section{Acknowledgments}

This study was supported by NIH Grant R01DA003371 (Dr. Fred Beauvais, Tri-Ethnic Center for Prevention Research, Department of Psychology, Colorado State University, Fort Collins, CO). The NIH had no further role in the manuscript design, the writing of the manuscript, or in the decision to submit the manuscript for publication.

\section{REFERENCES}

1. Swaim RC, Oetting ER, Edwards RW, et al. Links from emotional distress to adolescent drug use: A path model. J Consult Clin Psychol. 1989; 57:227-231. [PubMed: 2708609] 
2. Colder CR, Campbell RT, Ruel E, et al. A finite mixture model of growth trajectories of adolescent alcohol use: Predictors and consequences. J Consul Clin Psychol. 2002; 70:976-985.

3. Howard MO, Jenson JM. Inhalant use among antisocial youth: Prevalence and correlates. Addict Behav. 1999; 24:59-74. [PubMed: 10189973]

4. Evren C, Barut T, Saatcioglu O, et al. Axis I psychiatric comordidity among adult inhalant dependents seeking treatment. J Psychoactive Drugs. 2006; 38:57-64. [PubMed: 16681176]

5. Wu L, Howard MO. Psychiatric disorders in inhalant users: Results from The National Epidemiologic Survey on Alcohol and Related Conditions. Drug Alcohol Depend. 2007; 88:146155. [PubMed: 17129683]

6. Sakai JT, Hall SK, Milkulich-Gilbertson SK, et al. Inhalant use, abuse, and dependence among adolescent patients: Commonly comorbid problems. J Am Acad Child Adolesc Psychiatry. 2004; 43:1080-1088. [PubMed: 15322411]

7. Schinke SP, Fang L, Cole KCA. Substance use among early adolescent girls: Risk and protective factors. J Adolesc Health. 2008; 43:191-194. [PubMed: 18639794]

8. Khantzian EJ. Understanding addictive vulnerability. Neuro-Psychoanalysis. 2003; 5:5-21.

9. Hall DH, Queener JE. Self-medication hypothesis of substance use: Testing Khantzian's updated theory. J Psychoactive Drugs. 2007; 39:151-158. [PubMed: 17703709]

10. Dieterich SE, Stanley LR, Swaim RC, et al. Outcome expectancies, descriptive norms, and alcohol use: American Indian and white adolescents. J Prim Prev. 2013; 34:209-219. [PubMed: 23754535]

11. Skutle A. The relationship among self-efficacy expectancies, severity of alcohol abuse, and psychological benefits from drinking. Addict Behav. 1999; 24:87-98. [PubMed: 10189975]

12. Halper-Felsher BL, Biehl M, Kropp RY, et al. Perceived risks and benefits of smoking: differences among adolescents with different smoking experiences and intentions. Prev Med. 2004; 39:559_ 567. [PubMed: 15313096]

13. Morgan CJA, Noronha LA, Muetzelfeldt M, et al. Harms and benefits associated with psychoactive drugs: Findings of an international survey of active drug users. J Psychopharmacol. 2013; 27:497-506. [PubMed: 23438502]

14. Rimal RN, Real K. How behaviors are influenced by perceived norms: A test of the theory of normative social behavior. Communic Res. 2005; 32:389-414.

15. Storr CL, Westergaard R, Anthony JC. Early onset inhalant use and risk for opiate initiation by young adulthood. Drug Alcohol Depend. 2005; 78:253-261. [PubMed: 15893156]

16. Stanley LR, Harness S, Swaim RC, et al. Rates of substance use of American Indian students in 8th, 10th, and 12th grades living on or near reservations: Update, 2009-2012. Public Health Rep. 2014; 129:156-163. [PubMed: 24587550]

17. Oetting ER, Beauvais F. Adolescent drug use: Findings of national and local surveys. J Consult Clin Psychol. 1990; 58:385-394. [PubMed: 2212174]

18. McNamara RS, Swaim RC, Rosen LA. Components of negative affect as moderators of the relationship between early drinking onset and binge drinking. J Child Adolesc Subst Abuse. 2010; 19:108-121.

19. Swaim RC, Chen J, Deffenbacher JL, et al. Negative affect and alcohol use among non-Hispanic white and Mexican American adolescents. J Child Adolesc Subst Abuse. 2001; 11:55-75.

20. Swaim RC, Deffenbacher JL, Wayman JC. Concurrent and prospective effects of multidimensional aggression and anger on adolescent alcohol use. Aggress Behav. 2004; 30:356-372.

21. Swaim RC, Wayman JC. Multi-dimensional self-esteem and cross-cultural adolescent alcohol use: Concurrent and prospective effects. Am J Orthopsychiatry. 2004; 74:559-570. [PubMed: 15554815]

22. Whitesell NR, Mitchell CM, Kaufman CE, et al. Voices of Indian Teens Project Team. Developmental trajectories of personal and collective self-concept among American Indian adolescents. Child Dev. 2006; 77:1487-1503. [PubMed: 16999813]

23. Statham, A.; Rhoades, K. Gender and self-esteem: Narrative and efficacy in the negotiation of structural factors. In: Owens, TJ.; Stryker, S.; Goodman, N., editors. Extending self-esteem theory and research: Sociological and psychological currents. New York: Cambridge University Press; 2001. p. 255-284. 
24. Michaels ML, Barr A, Roosa MW, et al. Self-esteem: Assessing measurement equivalence in a multiethnic sample of youth. J Early Adolesc. 2007; 27:269-295.

25. Yoder KA, Whitbeck LB, Hoyt DR, et al. Suicidal ideation among American Indian youths. Arch Suicide Res. 2006; 10:177-190. [PubMed: 16574615]

26. Stumblingbear-Riddle G, Romans JSC. Resilience among urban American Indian adolescents: Exploration into the role of culture, self-esteem, subjective well-being, and social support. Am Indian Alsk Native Ment Health Res. 2012; 19:1-19. [PubMed: 22875470]

27. Thrane LE, Whitbeck LB, Hoyt DR, et al. Comparing three measures of depressive symptoms among American Indian adolescents. Am Indian Alsk Native Ment Health Res. 2004; 11:20-42. [PubMed: 15536587]

28. Shafer JL, Graham JW. Missing data: Our view of the state of the art. Psychol Methods. 2002; 7:147-177. [PubMed: 12090408]

29. Rubin, DB. Multiple imputation for non-response in surveys. New York: John Wiley \& Sons; 1987.

30. Bentler, PM.; Wu, JC. EQS 6. 1for Windows. Encino, CA: Multivariate Software Inc; 2010.

31. Satorra, A.; Bentler, PM. American Statistical Association 1988 Proceedings of the Business and Economic Sections. Alexandria, VA: American Statistical Association; 1988. Scaling corrections for chi square statistics in covariance structure analysis; p. 308-313.

32. Hu L, Bentler PM. Cutoff criteria for fit indexes in covariance structure analysis: Conventional criteria versus new alternatives. Struct Equ Modeling. 1999; 6:1-55.

33. Mulaik S. There is a place for approximate fit in structural equation modeling. Pers Individ Dif. 2007; 42:883-891.

34. Wu L, Howard MO, Pilowsky DJ. Substance use disorders among inhalant users: Results from the National Epidemiologic Survey on Alcohol and Related Conditions. Addict Behav. 2008; 33:968973. [PubMed: 18403132]

35. Hammond VL, Watson PJ, O'Leary BJ, et al. Preliminary assessment of Apache hopefulness: Relationships with hopelessness and with collective as well as personal self-esteem. Am Indian Alsk Native Ment Health Res. 2009; 16:42-51. [PubMed: 20052632]

36. Novins DK, Baron AE. American Indian substance use: The hazards for substance use initiation and progression for adolescents aged 14 to 20 years. J Am Acad Child Adolesc Psychiatry. 2004; 43:316-324. [PubMed: 15076265]

37. Storr CL, Westergaard R, Anthony JC. Early onset inhalant use and risk for opiate initiation by young adulthood. Drug Alcohol Depend. 78:253-261. [PubMed: 15893156] 


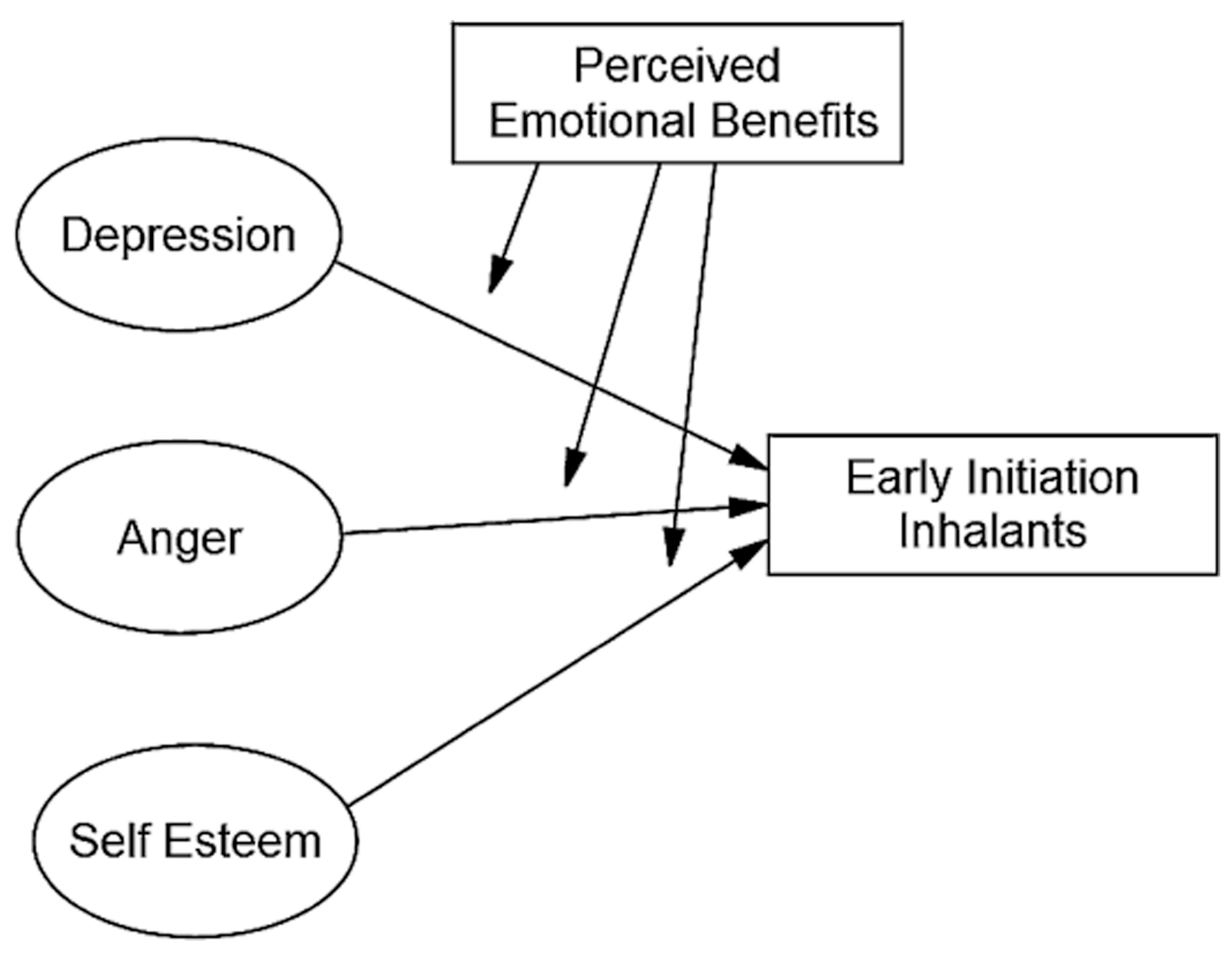

FIGURE 1.

Structural mode of Emotional Distress \& Inhalant Initiation. 

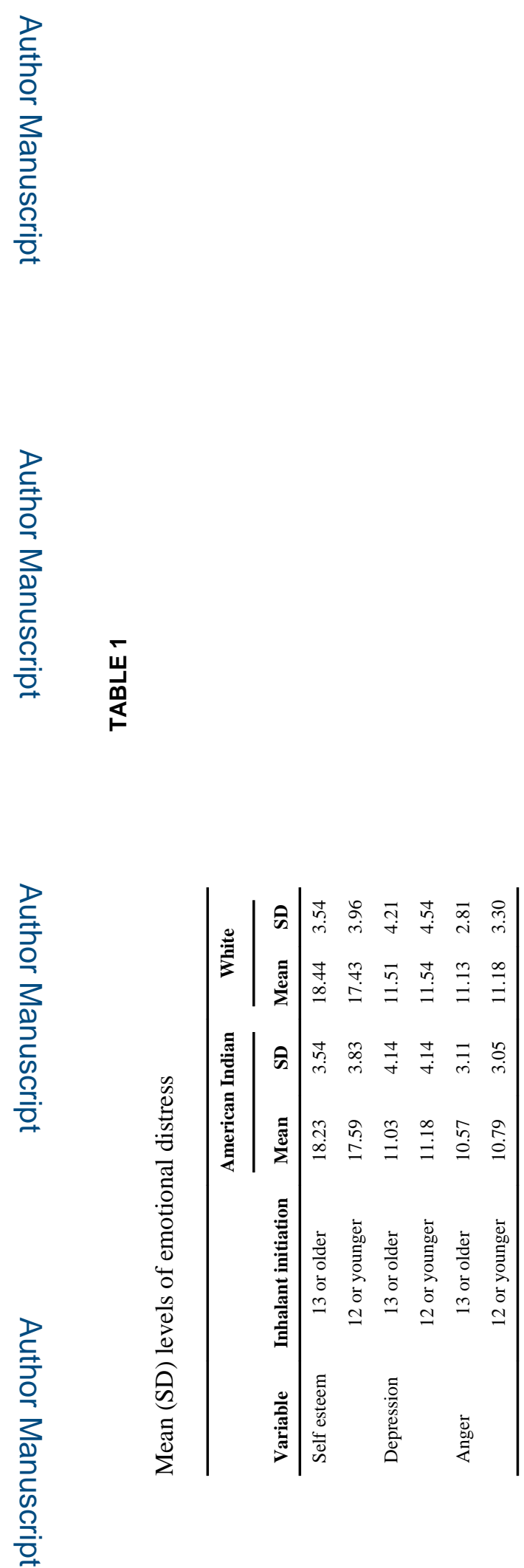

Am J Addict. Author manuscript; available in PMC 2015 December 22. 
